Internist 2010 $\cdot 51: 1069-1069$

DOI 10.1007/s00108-010-2683-5

Online publiziert: 15. Juli 2010

(c) Springer-Verlag 2010

\author{
V. Rosival ${ }^{1} \cdot$ T. zur Nieden ${ }^{2}$ \\ 1 SYNLAB, Abteilung für Laboratoriumsmedizin, Dérer Krankenhaus, Bratislava \\ ${ }^{2}$ Klinik für Kardiologie, Angiologie und Pneumologie, \\ Klinikum Esslingen, Esslingen am Neckar
}

\title{
Schnelle Blut-pH-Normalisierung bei komatösen Patienten mit Laktatazidose lebensrettend
}

\section{Meinungsaustausch zum Beitrag}

Zur Nieden T, Conrad T (2009) Komatöse Typ2-Diabetikerin mit metabolischer Azidose. Internist 50: 1276-1280

\section{Leserbrief}

\section{Rosival}

SYNLAB, Abteilung für Laboratoriumsmedizin, Dérer-Krankenhaus, Bratislava, Slowakei

Zur Nieden und Conrad [3] berichten über die erfolgreiche Behandlung von 2 komatösen Patientinnen mit schwerer Laktatazidose. Nach ihren Angaben ist die Sterblichkeit der Laktatazidose 50-80\% (S. 1278), die Patienten sterben im persistierenden Koma. In der ausführlichen Diskussion wurde jedoch von den Autoren die Problematik des tödlichen Komas kaum erörtert. Somit bleibt unklar, was von der umfangreichen Therapie für den lebensrettenden Erfolg ausschlaggebend war.

Die unmittelbare Ursache des Komas ist laut Edge et al. [2] niedriger Blut-pHWert (= hohe Konzentration von Wasserstoffionen $\mathrm{H}^{+}$). Zur Nieden und Conrad berichten zur metabolischen Azidose „Beeinträchtigt werden u. a. die Aktivität von Enzymen, Rezeptoren, Transmittern und Transportproteinen“" (S. 1279). Die Schlüsselrolle spielt dabei das glykolytische Enzym Phosphofruktokinase, das $\mathrm{pH}$-abhängig ist [7]: Mit sinkendem $\mathrm{pH}-$ Wert sinkt auch seine Aktivität und dadurch wird die Glukoseutilisation in den Gehirnzellen gehemmt bis unmöglich gemacht. Die klinischem Folgen von sinkendem Blut-pHWert sind deshalb Somnolenz - Sopor Koma - Tod im Koma. Bei beiden Patientinnen wurde der Blut-pH-Wert innerhalb von 10-14 h normalisiert (• Abb. 2). Es wird nicht genau berichtet, wann die $\mathrm{Pa}$ - tientinnen das normale Bewusstsein wiedererlangt haben, jedenfalls wäre es interessant zu erfahren, wie der Blut-pH-Wert zu diesem Zeitpunkt war.

Das Laktatanion an sich hat bei der Pathogenese des Komas keine Bedeutung: Weltweit werden Infusionen von Ringerlaktat verabreicht, ohne Berichte über Laktatintoxikation mit Bewusstseinsstörungen.

Abschließend kann man feststellen, dass bei komatösen Patienten mit Laktatazidose die lebensrettende Maßnahme die Normalisierung des niedrigen Blut$\mathrm{pH}$-Werts ist, egal ob es mit einem Dialyseverfahren erzielt wird (wie bei zur Nieden und Conrad) oder durch Infusionen von alkalisierenden Lösungen (wie z. B. [1]). Hauptsache ist, dass es so schnell wie möglich geschieht.

\section{Korrespondenzadresse \\ Dr. Viktor Rosival \\ SYNLAB, Abteilung für Laboratoriumsmedizin, \\ Dérer-Krankenhaus, Limbová 5 \\ 83305 Bratislava, Slowakei \\ rosivalv@hotmail.com}

\section{Erwiderung}

Die Anmerkungen von Dr. Rosival ergänzen die klinischen Fallberichte um wertvolle biochemische Details. Dr. Rosival konnte mit seinen Arbeiten in den vergangenen Jahren eindrücklich die Rolle des Komas sowie der umstrittenen Gabe von Bikarbonat bei der diabetischen Ketoazidose unterstreichen $[4,5,6]$.

Das Koma infolge der schweren Azidose muss als führendes klinisches Symptom auch im Falle unserer Berichte über Laktatazidosen unter Metformintherapie herausgestellt werden. Bei beiden Patientinnen wurde es jedoch von einem ein- drucksvollen Multiorganversagen begleitet, das Tage nach Ausgleich der Azidose hinaus anhielt.

Entscheidender Faktor der schweren Erkrankung ist die Azidose, deren Beseitigung oberste Therapiepriorität hat. Im Falle einer metformininduzierten Laktatazidose scheint die Hämodialyse einer alleinigen alkalisierenden Lösung [1] überlegen, da rasch sowohl Protonen als auch Metformin (als auslösende Noxe) selbst eleminiert werden.

Die Frage des Bewusstseinszustands unmittelbar nach Ausgleich der Azidose bleibt offen, da der kritisch instabile $\mathrm{Zu}$ stand in beiden Fällen eine rasche Extubation über mehrere Tage verhinderte.

\section{Korrespondenzadresse \\ Dr. Tim zur Nieden \\ Klinik für Kardiologie, Angiologie und Pneumologie Klinikum Esslingen, Hirschlandstraße 97 73730 Esslingen am Neckar tim.zurnieden@web.de}

\section{Literatur}

1. Ahmad S, Beckett M (2002) Recovery from ph 6.38: lactic acidosis complicated by hypothermia. Emerg Med J 19:169-171

2. Edge J, Roy Y, Bergomi A et al (2006) Conscious level in children with diabetic ketoacidosis is related to severity of acidosis and not to blood glucose concentration. Pediatr Diabetes 7:11-15

3. Nieden T zur, Conrad T (2009) Komatöse Typ-2-Diabetikerin mit metabolischer Azidose. Internist 50:12761280

4. Rosival V (2009) Acidosis, phosphofructokinase and diabetic coma. Br J Anaesth 102:279

5. Rosival V (2009) Sodium bicarbonate is beneficial in patients with diabetic ketoacidosis. Pediatr Crit Care Med 10:276

6. Rosival V (2009) Treating severe diabetic ketoacidosis with infusion of sodium bicarbonate. Bratisl Lek Listy 110:677

7. Trivedi B, Danforth WH (1966) Effect of pH on the kinetics of frog muscel phosphofructokinase. J Biol Chem 241:4110-4112 